\title{
Clinical implications of IgA rheumatoid factor subclasses
}

\author{
T Jónsson, $H$ Thorsteinsson, $\mathrm{S}$ Arinbjarnarson, J Thorsteinsson, $\mathrm{H}$ Valdimarsson
}

\begin{abstract}
Objectives-To evaluate the diagnostic and pathogenetic significance of $\operatorname{IgA}$ rheumatoid factor (RF) subclasses in rheumatoid arthritis (RA).

Methods-Rheumatoid factors of the IgA class and IgA1 and IgA2 subclasses were measured by enzyme linked immunosorbent assay in 58 patients with $R A, 31$ patients with other rheumatic diseases, 30 non-rheumatic individuals with increased concentrations of $\operatorname{IgA} R F$, and in 100 randomly selected healthy controls.

Results-Using a $95 \%$ cut off for the controls, $55 \%$ of the RA patients had increased total IgA RF, 64\% IgA1 RF, and $60 \%$ IgA2 RF. RA patients with extraarticular manifestations more often had increased concentrations of IgA RF and both subclasses than patients without such manifestations $(p \leqslant 0.01)$. Nearly all (31/32) RA patients with increased IgA RF had increases in both IgA RF subclasses, compared with $67 \%(20 / 30$ of nonrheumatic symptom free individuals with increased IgA RF $(p=0 \cdot 002)$.

Conclusion-Increased concentrations of the IgA2 RF subclass appears to be more specific for RA than increased IgA1 RF. Measurement of IgA RF subclasses may be clinically useful.
\end{abstract}

(Ann Rheum Dis 1995; 54: 578-581)

Rheumatoid factors (RF) are antibodies directed against the Fc part of immunoglobulin G (IgG) molecules. ${ }^{1}$ Small concentrations of RF can be detected in most healthy individuals, and these antibodies may have an immunoregulatory function. ${ }^{23}$ Increased concentrations of RF are characteristic of rheumatoid arthritis (RA), although increased $\mathrm{RF}$ can also be detected in some other rheumatic diseases, infections, and in a few apparently healthy individuals. ${ }^{145}$ Indeed, population studies have shown that the great majority of individuals with increased $\mathrm{RF}$, including IgA RF, do not have clinical manifestations of RA or other rheumatic diseases. ${ }^{56}$ Individual RF classes and subclasses can now be measured by solid phase assays. ${ }^{7-9}$

It has been reported that increased IgA RF is more specific for RA than are increases in other RF classes, and IgA RF has also been claimed to be an indicator of poor prognosis. ${ }^{6} 1011$ Thus IgA RF has been associated with early development of bone erosions ${ }^{12-14}$ and with extra-articular manifestations, especially from secretory glands and mucous membranes. ${ }^{1516}$ To our knowledge, measurement of IgA subclasses in RA patients has been reported previously only by Otten et $a l,{ }^{9}$ but their clinical significance was not evaluated.

We report the measurement of IgAl and IgA2 in a group of RA patients with and without extra-articular manifestations, patients with other rheumatic diseases, and in a group of symptom free individuals with increased total IgA RF.

\section{Patients and methods}

PATIENTS AND SAMPLES

We tested 219 serum samples for total IgA RF and both IgA RF subclasses. The samples were taken from three groups of patients: $58 \mathrm{RA}$ patients attending the University Hospital outpatient clinic; 31 patients with other rheumatic diseases, including 22 with systemic lupus erythematosus, three with mixed connective tissue disease, two with juvenile rheumatoid arthritis, three with psoriatic arthritis, and one with Reiter's disease; 30 non-rheumatic individuals with increased total IgA RF. As this was a hospital based study, the RA patients tended to have severe disease. Samples from 100 randomly selected healthy individuals, aged 31 to 50 , were used as controls.

The non-rheumatic individuals with increased concentrations of IgA RF had previously participated in a population based study on the significance of increased RF. ${ }^{6}$ When the blood samples were collected, all participants answered a structured questionnaire about past and present rheumatic symptoms and diseases known to be associated with increased RF. Furthermore, they were assessed clinically and radiographs taken of their hands for determination of bone erosions. The individuals selected for this analysis did not have symptoms or signs associated with rheumatic diseases.

MEASUREMENT OF TOTAL IgA RF, IgAI RF AND IgA2 RF

Total IgA RF activity was measured by an enzyme linked immunosorbent assay (ELISA) described previously. ${ }^{17}$ Briefly, microtitre plates (Dynatech Immulon I) were coated overnight at $4^{\circ} \mathrm{C}$ with a $40 \mu \mathrm{g} / \mathrm{ml}$ solution of purified rabbit IgG (Sigma), then serum samples $(1 / 40)$ and serial dilutions (1/40 to $1 / 2560$ ) of a local standard were incubated for two hours at room temperature. A $40 \mu \mathrm{g} / \mathrm{ml}$ solution of heat aggregated rabbit IgG was then added to block any free IgG binding sites on 

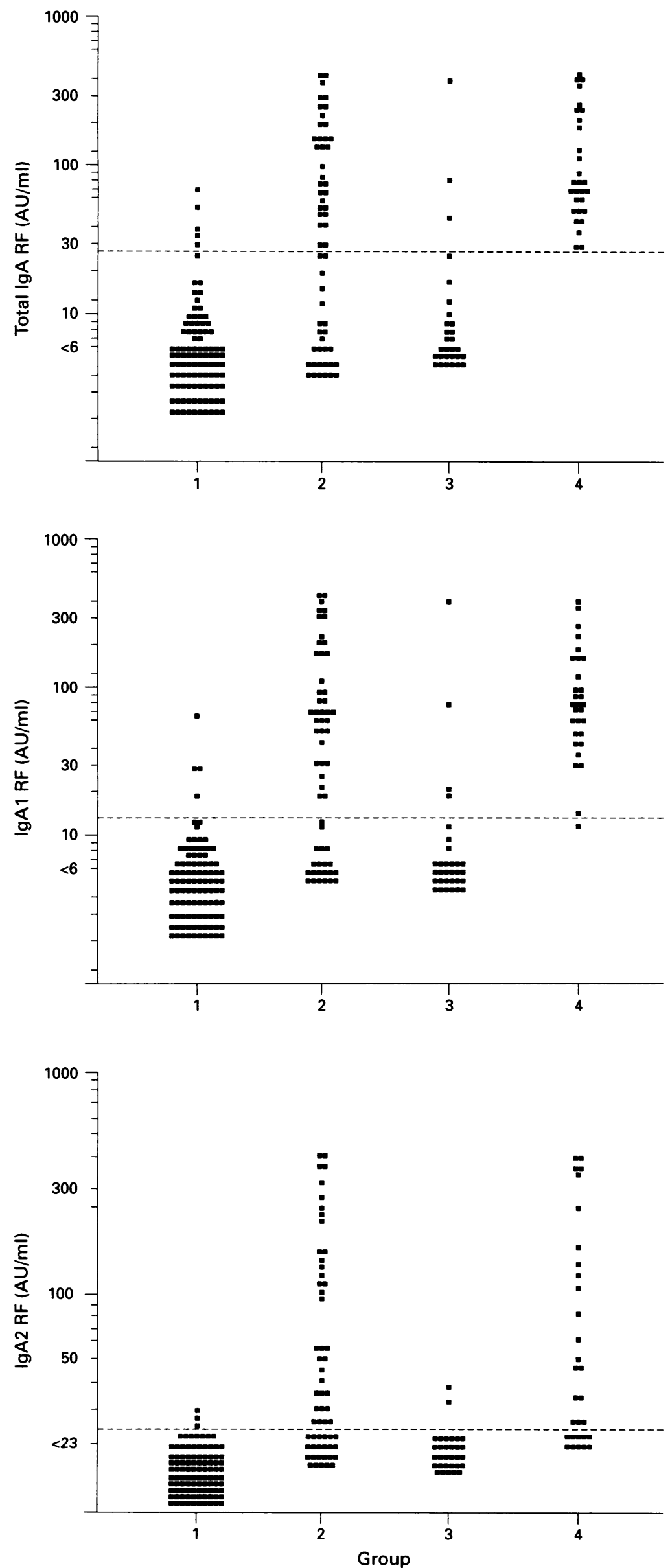

Total IgA rheumatoid factor (RF), IgA1 RF and IgA2 RF concentrations in patients and controls. Group $1=100$ randomly selected controls; group $2=58$ patients with $R A$; group $3=31$ patients with other rheumatic diseases; group $4=30$ non-rheumatic symptom free individuals with increased total $\operatorname{IgARF}$. The $95 \%$ cut off for the control group is indicated by the broken horizontal line. $A U=$ Arbitrary units. the solid phase bound RF. After two hours of incubation with mouse monoclonal antihuman IgA (Oxoid, clone 2D7), an appropriate dilution of alkaline phosphatase conjugated rabbit anti-mouse IgG was added. Finally, a $1 \mathrm{mg} / \mathrm{ml}$ solution of para-nitrophenylphosphate substrate solution was incubated and the colour reaction read in a microplate reader (Flow Laboratories) when absorbance of the $1 / 40$ dilution of the local standard was approximately $1 \cdot 6-1 \cdot 8$. Results were expressed in arbitrary units (AU/ml) according to a local standard (see below). IgAl $\mathrm{RF}$ and IgA2 RF were measured in the same manner, except that mouse monoclonal antiIgA1 (Nordimmune, clone NI 69-11) or antiIgA2 (Nordimmune, clone NI-512) antibodies were used.

A local standard was prepared from a serum pool collected from 11 patients with RA and large concentrations of RF. This standard was titrated against the International Reference Preparation of Rheumatoid Arthritis Serum (Statens Serum Institute, Copenhagen, Denmark). The local standard was found to contain 3.75 times more of total IgA RF than the International Standard and was therefore given an arbitrary value of 375 units (AU/ml). Similarly, the local standard was given a value of $375 \mathrm{AU} / \mathrm{ml}$ for both IgA RF subclasses.

RF values above the $95 \%$ cut off level for 100 randomly selected controls were considered to be increased. Thus the upper limit of normal was $25 \mathrm{AU} / \mathrm{ml}$ for total IgA RF activity, $11 \mathrm{AU} / \mathrm{ml}$ for IgA1 RF, and $23 \mathrm{AU} / \mathrm{ml}$ for IgA2 RF.

\section{MEASUREMENT OF IgM RF}

IgM RF was measured by ELISA as previously described for IgA RF, except that mouse monoclonal anti-human IgM antibodies (Sigma, clone MB-11) were used for detection of the solid phase bound RF. ${ }^{17}$ Fewer than 5\% of randomly selected controls were positive for IgM RF by this method.

STATISTICAL EVALUATION

The results of the study were evaluated with the chi-square test and Spearman's rank coefficient of correlation. The level of significance was set at $\mathrm{p}<0.05$.

\section{Results}

The figure shows the concentrations of $\operatorname{IgA}$ $\mathrm{RF}$, IgA1 RF, and IgA2 RF in the patients and control subjects. Using a $95 \%$ cut off for the healthy controls, increased concentrations of IgA RF were found in $55 \%(32 / 58)$ of the RA patients; $64 \%$ (37/58) had increased IgAl RF, and $60 \%(35 / 58)$ had increased IgA2 RF. In contrast, only $10 \%(3 / 31)$ of the patients with other rheumatic diseases had increased IgA RF; $13 \%$ (4/31) had increased IgA1 RF and $6 \%(2 / 31)$ had increased IgA2 RF $(\mathrm{p}<0.0001)$.

Table 1 shows the prevalence of increased IgM RF, IgA RF, and both IgA RF subclasses 
Table 1 Patients with increased IgM rheumatoid factor (RF), IgA RF, and its subclasses in patients with rheumatoid arthritis $(R A)$ and other rheumatic diseases

\begin{tabular}{llllll}
\hline Increase in & \multicolumn{2}{l}{$R A(n=58)$} & & \multicolumn{2}{c}{$\begin{array}{c}\text { Other rheumatic } \\
\text { diseases }(n=31)\end{array}$} \\
\cline { 2 - 3 } \cline { 6 - 6 } \cline { 5 - 6 } & No & $\%$ & & No & $\%$ \\
\hline IgM RF & 40 & 69 & & 8 & 26 \\
IgA RF & 32 & 55 & & 3 & 10 \\
IgA1 RF & 37 & 64 & & 4 & 13 \\
IgA2 RF & 35 & 60 & & 2 & $6^{\star}$ \\
\hline
\end{tabular}

${ }^{\star} \mathrm{p}=0.038$ compared with IgM RF.

in patients with $\mathrm{RA}$ and other rheumatic diseases. Among these patients, 26\% (8/31) had increased IgM RF, while only $6 \%(2 / 31)$ had increased IgA2 RF ( $p=0.038)$.

Twenty five of the 58 RA patients $(43 \%)$ had some extra-articular manifestations, including 21 with rheumatoid nodules, seven with sicca symptoms, two with rheumatoid lung, one patient with vasculitis and one with Felty's syndrome. Compared with the RA patients who did not have extra-articular manifestations, significantly greater numbers of these patients had increased concentrations of IgA RF $(p=0.006)$, IgA1 RF $(p=0.010)$, and IgA2 RF ( $p=0.008$ ) (table 2). This difference was not attributable to the RA patients with sicca syndrome, as they did not have greater concentrations of IgA RF or its subclasses than patients with other extra-articular manifestations (data not shown).

Table 3 shows that all RA patients with increased total IgA RF had also increased concentrations of the IgAl RF subclass, and 97\% (31/32) had increased IgA2 RF. Of the non-rheumatic individuals with increased total IgA RF, 97\% (29/30) had increased IgA1 RF, but only $67 \%(20 / 30)$ had increased IgA2 RF. Thus $97 \%(31 / 32)$ of the IgA RF positive RA patients had increases in both IgA RF subclasses, while $33 \%(10 / 30)$ of non-rheumatic individuals with increased total IgA RF had an isolated increase in IgA1 RF ( $p=0.002)$. The non-rheumatic individuals with increased IgA2

Table 2 Rheumatoid arthritis $(R A)$ patients with and without extra-articular manifestations (EAM) in relation to increased concentrations of IgA rheumatoid factor $(R F)$ and its subclasses

\begin{tabular}{|c|c|c|c|c|c|}
\hline \multirow[t]{3}{*}{ Increase in } & \multicolumn{4}{|c|}{$R A$ patients } & \multirow[t]{3}{*}{$p \dagger$} \\
\hline & \multicolumn{2}{|c|}{$\begin{array}{l}\text { With EAM } \\
(n=25)\end{array}$} & \multicolumn{2}{|c|}{$\begin{array}{l}\text { Without EAM } \\
(n=33)\end{array}$} & \\
\hline & No & $\%$ & No & $\%$ & \\
\hline $\begin{array}{l}\text { Total IgA RF } \\
\text { IgA1 RF } \\
\text { IgA2 RF }\end{array}$ & $\begin{array}{l}19 \\
21 \\
20\end{array}$ & $\begin{array}{l}76 \\
84 \\
80\end{array}$ & $\begin{array}{l}13 \\
17 \\
15\end{array}$ & $\begin{array}{l}39 \\
52 \\
45\end{array}$ & $\begin{array}{l}0.006 \\
0.010 \\
0.008\end{array}$ \\
\hline
\end{tabular}

†Evaluated with the chi-square test.

Table 3 Increases in concentrations of IgA rheumatoid factor (RF) subclasses in patients with rheumatoid arthritis $(R A)$ and non-rheumatic symptom free individuals with increased total IgA RF

\begin{tabular}{llllllll}
\hline Increase in & \multicolumn{2}{l}{$R A(n=32)$} & & \multicolumn{2}{l}{$\begin{array}{l}\text { Non-rheumatic } \\
\text { individuals }(n=30)\end{array}$} & p† \\
\cline { 2 - 3 } & No & $\%$ & & No & $\%$ & \\
\hline IgA1 RF & 32 & 100 & & 29 & 97 & NS \\
IgA2 RF & 31 & 97 & & 20 & 67 & 0.002 \\
\hline
\end{tabular}

tEvaluated with the chi-square test.

NS $=$ Not significant.
Table 4 Correlation between IgA rheumatoid factor $(R F)$ and its subclasses in patients with rheumatoid arthritis $(R A)$ and non-rheumatic symptom free individuals with increased total $\operatorname{Ig} A R F$

\begin{tabular}{|c|c|c|c|c|}
\hline & \multicolumn{2}{|l|}{$R A$} & \multicolumn{2}{|c|}{$\begin{array}{l}\text { Non-rheumatic } \\
\text { individuals }\end{array}$} \\
\hline & Correlation $\dagger$ & $p$ & Correlation & $p$ \\
\hline $\begin{array}{l}\text { Total IgA RF } v \text { IgA1 RF } \\
\text { Total IgA RF } v \text { IgA2 RF } \\
\text { IgA1 RF } v \text { IgA2 RF }\end{array}$ & $\begin{array}{l}0.853 \\
0.629 \\
0.736\end{array}$ & $\begin{array}{l}0.0001 \\
0.0005 \\
0.0001\end{array}$ & $\begin{array}{l}0.828 \\
0.206 \\
0.352\end{array}$ & $\begin{array}{l}0.0001 \\
\text { NS } \\
\text { NS }\end{array}$ \\
\hline
\end{tabular}

†Results are given as Spearman's coefficient of correlation (Rho). NS $=$ Not significant .

RF did not have greater concentrations of IgA RF than those who did not have increased IgA2 RF (data not shown).

In addition, a significant positive correlation was noted between total IgA RF and both subclasses in patients with RA, while in the non-rheumatic individuals there was a positive correlation only between IgA RF and IgA1 RF (table 4).

\section{Discussion}

Although increased RF, as measured by conventional agglutination tests, is found in the majority of RA patients, it is also frequently found in patients with other rheumatic and non-rheumatic diseases. Agglutination tests mainly detect IgM RF and do not discriminate between patients with isolated increases in IgM $\mathrm{RF}$ and those with increases in both IgM RF and IgA RF. We have previously demonstrated that a concomitant increase in concentrations of IgM RF and IgA RF is much more specific for RA than increase in IgM RF or IgG RF alone. ${ }^{18}$

This study indicates that increased IgA2 RF is markedly more common among RA patients than among patients with other rheumatic diseases, or symptom free individuals with increased total IgA RF. Furthermore, increased IgA2 RF seems to be more specific for RA than increased IgM RF (table 1).

Otten et $a l^{9}$ reported a prevalence of increased IgA1 RF in RA patients similar to the one we observed in this study $(73 \% v 64 \%)$. However, they detected increased IgA2 RF concentrations in only $36 \%$ of their patients, compared with $60 \%$ of the RA patients in our study. This cannot be explained by different criteria used for defining the upper limit of normal, as a $95 \%$ cut off was used in both studies. No clinical details are presented in the report by Otten et al and it is therefore possible that their patients had less severe disease; however, $45 \%$ of our patients who had no evidence of extra-articular manifestations had increased IgA2 RF. A more likely explanation for the discrepancy may be that Otten's group used human IgG as antigen in their ELISA system, while we used rabbit IgG. It has been claimed that a stronger association has been found between increased IgA RF and bone erosions when rabbit IgG, rather than human IgG, has been used as antigen. ${ }^{19}$ The pathophysiological significance of this phenomenon is not known, but it is conceivable that IgG molecules in individuals susceptible to $R A$ 
share epitope(s) with rabbit IgG. It has indeed been shown that RF reacts more strongly with IgG from RA patients than with IgG from healthy subjects. ${ }^{20}$

Otten et al did not analyse the relationship between IgA RF subclasses and disease manifestations in RA. ${ }^{9}$ Our findings that RA patients with extra-articular manifestations had greater concentrations of both IgA RF subclasses than patients without such manifestations are consistent with several previous studies, ${ }^{15162122}$ but also show that there is no real difference between the subclasses in this respect.

It has been reported that increase in RF concentration often precedes the clinical onset of RA by months or even years, and this may be particularly true for increases in IgA RF. $^{62324}$ Thus it is possible that healthy individuals with increases in both IgA RF subclasses are at greater risk of later developing RA than those individuals who have increases in only the IgA1 RF subclass. It is intended to follow the IgA RF positive non-rheumatic individuals presented in this study in order to evaluate this possibility.

This study has indicated that measurement of IgA RF subclasses may be diagnostically useful, but larger prospective studies are required to elucidate fully the prognostic and pathogenic significance of increased IgAl and IgA2 RF.

The authors wish to thank Kristján Steinsson, Helgi Jonsson, Arni Jón Geirsson, and Kristján Erlendsson at the National University Hospital, Landspitalinn, Reykjavik for their assistance. This study was supported by a Syntex rheumatology grant.

1 Egeland T, Munthe E. Rheumatoid factors. Clin Rheum Dis 1983; 9: 135-60.

2 Sinclair N R, Panoskaltis A. Immunoregulation by Fc signals: a mechanism for self-nonself discrimination. Immunol Today 1987; 8: 76-9.

3 del Guerco P. Regulatory structures on the $V$ and $C$ regions of immunoglobulin molecules. Immunol Today 1987; 8: 304-8.

4 Thorsteinsson J, Björnsson O J, Kolbeinsson A, Allander E, Sigfússon $\mathrm{N}$, Ólafsson $\mathrm{O}$. A population study of rheumatoid factor in Iceland. A 5 year follow-up of 50 women with rheumatoid factor (RF). Ann Clin Res 1975; 7: 183-94.
5 Mikkelsen D W, Dodge H J, Duff I F, Kato H. Estimates on the prevalence of rheumatic diseases in the population of Tecumseh, Michigan, 1959-60. f Chron Dis 1967; 20: of Tecurnse.

6 Jónsson T, Thorsteinsson J, Kolbeinsson A, Jónasdóttir E, Sigfússon N, Valdimarsson H. Population study of the importance of rheumatoid factor isotypes in adults. Ann Rheum Dis 1992; 51: 863-8.

7 Carson D A, Lawrance S, Catalano M A, Vaughan J H, Abraham G. Radioimmunoassay of IgG and IgM rheumatoid factors reacting with human IgG. $f$ Immunol 1977; 119: 295-300.

8 Teitsson I, Valdimarsson $\mathrm{H}$. Use of monoclonal antibodies and $F\left(a b^{\prime}\right) 2$ enzyme conjugates in ELISA for IgM, IgG and IgA rheumatoid factors. $₹$ Immunol Methods 1984; 71 : 149-61.

9 Otten G, Mohamed R D, Jacob M, Hennie H, Breedveld $F$ C. Subclass distribution and size of human IgA rheumatoid factor at mocosal and nonmucosal sites. Arthritis Rheum 1991; 34: 831-9.

10 Jónsson $T$, Valdimarsson $H$. Clinical significance of rheumatoid factor isotypes in serpositive arthritis. Rheumatol Int 1992; 12: 111-3.

11 Teitsson I. IgA rheumatoid factor as predictor of disease activity. Scand 7 Rheumatol 1988; 17 (suppl 75): 233-7.

12 Teitsson I, Withrington $\mathrm{R} \mathrm{H}$, Seifert $M \mathrm{H}$, Valdimarsson $\mathrm{H}$. Prospective study of early rheumatoid arthritis. I. Prognostic value of IgA rheumatoid factor. Ann Rheum Dis 1984; 43: 673-8.

13 Arnason J A, Jónsson T, Brekkan A, Sigurjónsson K, Valdimarsson $\mathrm{H}$. Relation between bone erosions and rheumatoid factor isotypes. Ann Rheum Dis 1987; 46: rheumat

14 Winska W H, Thompson K, Young A, Corbett M, Shipley $M$, Hay F. IgA and IgM rheumatoid factors as markers of later erosive changes in rheumatoid arthritis (RA). Scand 7 Rheumatol 1988; 17 (suppl 75): 238-43.

15 Dunne J V, Carson D A, Spiegelberg H L, Alspaugh M A, Vaughan J H. IgA rheumatoid factor in the sera and saliva of patients with rheumatoid arthritis and Sjögren's syndrome. Ann Rheum Dis 1979; 38: 161-5.

16 Lúoviksson B R, Jónsson T, Erlendsson K, Sigfússon Á. Disease manifestations in patients with isolated elevation of IgA reumatoid factor. Scand $\mathcal{f}$ Rheumatol 1992; 21: of IgA

17 Jónsson T, Árnason J A, Valdimarsson H. Enzyme-linked immunosorbent assay (ELISA) screening test for detection of rheumatoid factor. Rheumatol Int 1986; 6: 199-204

18 Jónsson T, Valdimarsson H. Prognostic value of rheumatoid factors in rheumatoid arthritis. $\mathcal{F}$ Rheumatol. In press.

19 Jónsson T, Valdimarsson H. Is measurement of rheumatoid factor isotypes clinically useful? Ann Rheum Dis 1993; 52: $161-4$

20 Carter S D, Makh S R, Ponsford F M, Elson C J. Rheumatoid factor has increased reactivity with IgG from Rheumatoid factor has increased reactivity with IgG from synovial fluids of patients with rheumatoid arth

21 Tarkowski A, Nilsson L- $\AA$. Isotype-specific measurement of rheumatoid factor with reference to clinical manifestations of rheumatoid arthritis. $f$ Clin Lab Immunol 1983; 12: 129-35.

22 Westedt M L, Herbrink P, Molenaar J L, et al. Rheumatoid factors in rheumatoid arthritis and vasculitis. Rheumatol Int 1985; 5: 209-14.

23 Del Puente A, Knowler W C, Pettitt D J, Bennett P H. The incidence of rheumatoid arthritis is predicted by rheumatoid factor titer in a longitudinal population study. Arthritis Rheum 1988; 31: 1239-44.

24 Tuomi T, Palosuo T, Aho K. The distribution of classspecific rheumatoid factors is similar in rheumatoid and pre-illness sera. Scand $\mathcal{I}$ Immunol 1986; 24: 751-4. 